\title{
Heat and Mass Transfer in MHD Micropolar Fluid Flow over a Stretching Sheet with Velocity and Thermal Slip Conditions
}

\author{
E. O. Fatunmbi' A. Adeniyan² \\ ${ }^{1}$ Department of Mathematics and Statistics, Federal Polytechnic, Ilaro, Nigeria \\ ${ }^{2}$ Department of Mathematics, University of Lagos, Akoka, Nigeria \\ Email: *olusojiephesus@yahoo.com, aadeniyan@unilag.edu.ng
}

How to cite this paper: Fatunmbi, E.O. and Adeniyan, A. (2018) Heat and Mass Transfer in MHD Micropolar Fluid Flow over a Stretching Sheet with Velocity and Thermal Slip Conditions. Open Journal of Fluid Dynamics, 8, 195-215.

https://doi.org/10.4236/ojfd.2018.82014

Received: April 1, 2018

Accepted: June 26, 2018

Published: June 29, 2018

Copyright $\odot 2018$ by authors and Scientific Research Publishing Inc. This work is licensed under the Creative Commons Attribution International License (CC BY 4.0).

http://creativecommons.org/licenses/by/4.0/

\begin{abstract}
This study presents the problem of a steady, two-dimensional, heat and mass transfer of an incompressible, electrically conducting micropolar fluid flow past a stretching surface with velocity and thermal slip conditions. Also, the influences of temperature dependent viscosity, thermal radiation and non-uniform heat generation/absorption and chemical reaction of a general order are examined on the fluid flow. The governing system of partial differential equations of the fluid flow are transformed into non-linear ordinary differential equations by an appropriate similarity variables and the resulting equations are solved by shooting method coupled with fourth order Runge-Kutta integration scheme. The effects of the controlling parameters on the velocity, temperature, microrotation and concentration profiles as well as on the skin friction, Nusselt number, Sherwood and wall couple stress are investigated through tables and graphs. Comparison of the present results with the existing results in the literature in some limiting cases shows an excellent agreement.
\end{abstract}

\section{Keywords}

Micropolar Fluid, Magnetohydrodynamics, Slip Conditions, Stretching Sheet

\section{Introduction}

In the recent years, the study of non-Newtonian fluids has attracted considerable attention from researchers due to its increasing usefulness and practical relevance in many industrial processes. These fluids are particularly important in real industrial applications, such as in polymer engineering, crude oil extraction, 
food processing etc. It has been observed that the Navier-Stokes equations of classical hydrodynamic cannot adequately describe the complex rheological behaviour that fluids of industrial significance exhibit at micro and nano scales. These have led to the development of various microcontinuum theories such as simple microfluids, simple deformable directed fluids, polar fluids, anisotropic fluids and micropolar fluids depending on different physical characteristics. However, as a result of diverse fluid characteristics in nature, all the non-Newtonian fluids cannot be captured by a single constitutive model, hence, different models of non-Newtonian fluids have been formulated such as Casson fluid, Jeffery fluid, Maxwell fluid, Ostwald de-Waele power law fluid and Micropolar fluids (Chen et al. [1]).

Holt and Fabula [2], Vogel and Patterson [3] in their experiments on fluids with small amount of polymeric additives discovered that these fluids manifest a reduction in skin friction near a rigid body. In consequence, Eringen [4] [5] formulated the theory of micropolar fluids and as well derived the constitutive equations for the theory of thermo-micropolar fluids. Micropolar fluids are important branch of non-Newtonian fluids dynamics with microstructure and constitute a substantial generalization of the Navier-Stokes model. By this theory, each element of the fluid is associated to two sets of degrees of freedom: translatory degrees of freedom, giving rise to velocity, and rotation/stretch, allowing the particles to undergo independent intrinsic spins and homogenous deformation (Eringen [5]). These fluids provide a mathematical model for investigating many complicated and complex fluids motions such as suspension solution, blood rheology and colloidal fluids. The presence of micropolar fluids can be found in polymeric fluids, fluid suspensions, animal blood, liquid crystals, colloidal fluids, clouds with dust etc. (Ahmadi [6]; Hayat et al. [7]). Its applications in a number of industrial processes, such as extrusion of polymer, the flow of exotic lubricants, colloidal suspensions and the cooling of metallic plate in water bath have also boosted the interest of researchers in studying it (Rahman [8]). A detailed review, on the theory and applications of micropolar fluids, was given by Lukaszewicz [9].

The study of flow and heat transfer induced by stretching surfaces plays a vital role in industrial and engineering processes. For instance, the aerodynamic extrusion of plastic sheets, wire drawing, glass fiber production, paper production and hot rolling. The pioneering work on the steady, boundary layer flow of an incompressible viscous fluid induced by linearly stretching sheet was carried out by Crane [10]. Gupta and Gupta [11] extended the work to include heat and mass transfer on stretching sheet with suction or blowing.

The inclusion of the magnetic field on the study of flow and heat transfer past stretching plates has practical applications in engineering activities, for instance, hot rolling, the extrusion of polymer sheet from a die and the cooling of metallic sheets. In order to achieve top-grade property of the final product during the fabrication processes, the rate of heating and cooling can be controlled by the use of electrically conducting fluid and the application of magnetic field (Mukho- 
padhyay [12]). To this end, Eldabe et al. [13] studied MHD flow of a micropolar fluid past a stretching sheet with heat transfer. Elbashbeshy and Bazid [14] examined heat transfer over a stretching sheet embedded in a porous medium. Kumar [15] numerically studied the problem of heat and mass transfer in a hydromagnetic flow of a micropolar fluid past a stretching sheet using the Finite element technique. The author reported that the fluid velocity increased with a rise in the material parameter, microrotation and temperature also rise with an increase in the magnetic field parameter while the velocity falls with an increase in the magnetic field parameter.

The study of heat and mass transfer analysis with chemical reaction, heat generation/absorption in the boundary layer flow is of practical importance due their importance in chemical processes and hydrometallurgical industries, for instance, food processing, manufacturing of ceramics and polymer production (Das [16]; Mishra et al. [17]). Also, heat generation/absorption influence may change the temperature distribution of the fluid flow and in consequence affect various engineering devices. To this end, Bhattacharyya and Layek [18] studied MHD boundary layer flow with diffusion and chemical reaction over a porous flat plate with suction/blowing. Mohammed and Abo-Dahab [19] examined heat and mass transfer in MHD micropolar flow over a vertical moving porous plate in a porous medium with heat generation using perturbation technique. The authors reported that the translational velocity across the boundary layer and the magnitude of microrotation at the wall are decreased with an increase in the values of magnetic and Prandtl parameters while the trend was reversed with an increase in the values of thermal radiation. Other researchers who have studied boundary layer flow with heat sources include (Olajuwon et al. [20]; Pal and Chatterjee [21]).

Many researchers assumed constant fluid properties, however, physical properties of fluid may change largely with temperature, especially fluid viscosity. The increase in temperature enhances the transport phenomena by decreasing the viscosity across the momentum boundary layer such that the rate of heat transfer at wall is affected, similarly, a decrease in fluid viscosity can make the fluid velocity decrease appreciably with an increase in transverse distance from a stretching plate. Thus, to accurately predict the flow behaviour, adequate attention should be paid to the variation of viscosity with temperature. Pal and Mondal [22] investigated the influence of temperature-dependent viscosity and variable thermal conductivity on Newtonian fluid. Bhattacharyya et al. [23] investigated boundary layer slip flow and heat transfer past a stretching sheet with temperature dependent viscosity.

The influence of thermal radiation heat transfer is significant on various flow because many engineering processes happen at high temperature, and the knowledge on radiation heat transfer becomes useful for the design of pertinent equipment, nuclear power plants, various propulsion devices, space technology and high temperature processes such as polymer processing industry where the quality of the end product depends to some extent on the heat controlling fac- 
tors. The above researchers have limited their investigations to flow and heat transfer problems of Newtonian/non-Newtonian fluids under the assumption of no-slip boundary condition (i.e. the assumption that the fluid adheres to the boundary surface). However, in some practical situations, this assumption does not hold and it may be necessary to replace the no-slip boundary condition with the partial slip boundary condition for some practical flow problems. The non-adherence of fluid to a solid boundary is known as velocity slip. The slip and temperature jump boundary conditions represent a discontinuity in the transport variable across the interface and describes more accurately the non-equilibrium region near the surface. Slip flow problems are very essential on both the stationary and moving boundary as there are various Newtonian and non-Newtonian fluids such as particulate fluids e.g. emulsions, suspensions and polymer solutions in which there may be a slip between the fluid and the boundary (Wang [24]). The applications of such study in technology can be found in the polishing of artificial heart valves and internal cavities (Mukhopadhyay [25]). To this end, Anderson [26] examined the slip-flow of a Newtonian fluid over a linearly stretching sheet. Das [16] investigated slip effects on heat and mass transfer in MHD micropolar fluid flow over an inclined plate with thermal radiation and chemical reaction. Devi et al. [27] examined radiation effect on MHD slip flow past a stretching sheet with variable viscosity and heat source/sink. Kemparaju et al. [28] investigated heat transfer in MHD Newtonian fluid flow over a stretching sheet with velocity and thermal slip conditions.

The aim of this study is to examine the influence of both the velocity and thermal slip on heat and mass transfer in MHD micropolar fluid flow over a stretching sheet under the influence of variable fluid viscosity, thermal radiation, non-uniform heat source/sink and homogeneous chemical reaction of a general order. The nonlinear partial differential equations governing the flow are transform into nonlinear ordinary differential equations by an appropriate similarity transformations variables while the resulting equations are solved by applying the fourth order Runge-Kutta integration scheme.

\section{Problem Formulation}

Consider a laminar, boundary layer slip flow of a viscous, incompressible, MHD micropolar fluid past a flat stretching sheet. The cartesian coordinate system is $(\bar{x}, \bar{y}, \bar{z})$ and the corresponding velocity components are $(\bar{u}, \bar{v}, 0)$. The $x$-axis is directed towards the continuous stretching sheet along the flow while the $y$-axis is normal to it. The stretching velocity is assumed to be $\bar{u}_{w}=a \bar{x}$ while the velocity upstream is assumed to be zero, the temperature and concentration of the sheet are $T_{w}$ and $C_{w}$ respectively. The flow is confined to the region $y>0$. A transverse magnetic field $\boldsymbol{B}=\left(0, B_{o}, 0\right)$ of strength $B_{o}$ is applied normal to the flow direction as displayed in Figure 1. Also, the angular velocity $\boldsymbol{\omega}=\left(\omega_{1}, \omega_{2}, \omega_{3}\right)=(0,0, N(\bar{x}, \bar{y}))$ is assumed. The radiative heat flux term in $\bar{x}$ direction is considered negligible as compared to that in the $\bar{y}$ direction. It is assumed also that the magnetic Reynolds number is sufficiently small such that 


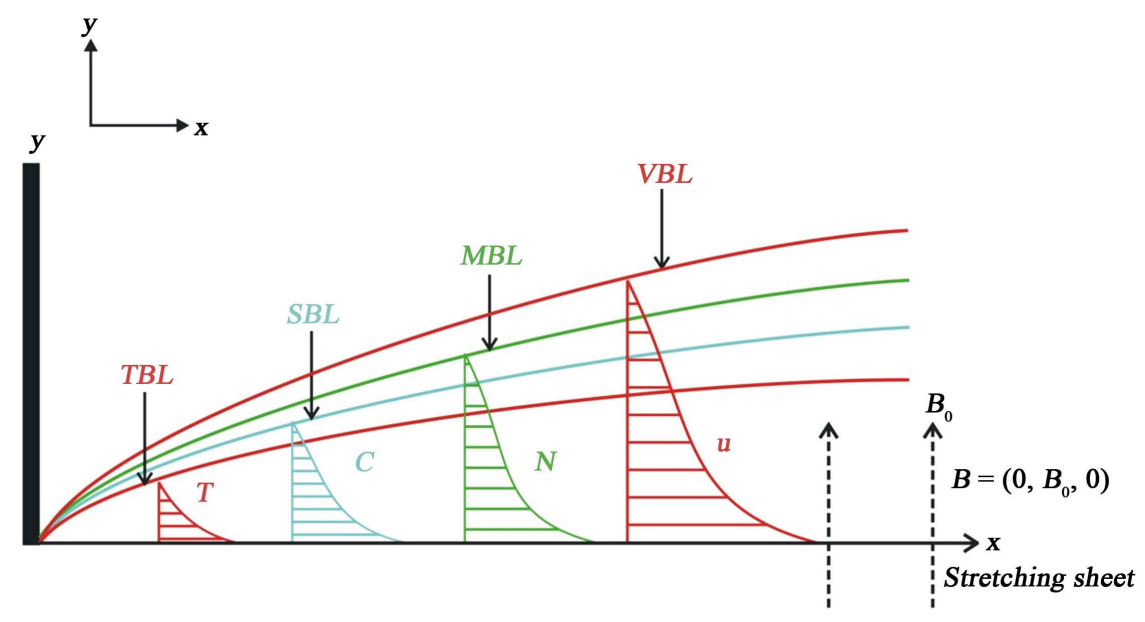

Figure 1. Geometry of the flow.

the induced magnetic field is negligible as compared to the applied magnetic field.

Under the stated assumptions and the boundary layer approximations, the governing boundary layer continuity, momentum, microrotation and energy are respectively given as:

$$
\begin{gathered}
\frac{\partial \bar{u}}{\partial \bar{x}}+\frac{\partial \bar{v}}{\partial \bar{y}}=0 \\
\bar{u} \frac{\partial \bar{u}}{\partial \bar{x}}+\bar{v} \frac{\partial \bar{u}}{\partial \bar{y}}=\frac{1}{\rho} \frac{\partial}{\partial \bar{y}}\left(\mu(\bar{T}) \frac{\partial \bar{u}}{\partial \bar{y}}\right)+\frac{\kappa}{\rho} \frac{\partial^{2} \bar{u}}{\partial \bar{y}^{2}}+\frac{\kappa}{\rho} \frac{\partial \bar{N}}{\partial \bar{y}}-\frac{\sigma B_{o}^{2}}{\rho} \bar{u} \\
\bar{u} \frac{\partial \bar{N}}{\partial \bar{x}}+\bar{v} \frac{\partial \bar{N}}{\partial \bar{y}}=\frac{\gamma}{\rho j} \frac{\partial^{2} \bar{N}}{\partial \bar{y}^{2}}-\frac{\kappa}{\rho j}\left(2 \bar{N}+\frac{\partial \bar{u}}{\partial \bar{y}}\right), \\
\bar{u} \frac{\partial \bar{T}}{\partial \bar{x}}+\bar{v} \frac{\partial \bar{T}}{\partial \bar{y}}=\frac{k}{\rho C_{p}} \frac{\partial^{2} \bar{T}}{\partial \bar{y}^{2}}-\frac{1}{\rho C_{p}} \frac{\partial q_{r}}{\partial \bar{y}}+\frac{q^{\prime \prime \prime}}{\rho c_{p}}, \\
\bar{u} \frac{\partial C}{\partial \bar{x}}+\bar{v} \frac{\partial C}{\partial \bar{y}}=D m \frac{\partial^{2} C}{\partial \bar{y}^{2}}-k_{r}\left(\bar{C}-C_{\infty}\right)^{n}
\end{gathered}
$$

The associated boundary conditions for Equations (1)-(5) are:

$$
\begin{aligned}
& \bar{y}=0: \bar{u}=u_{w}+\lambda \frac{\partial \bar{u}}{\partial \bar{y}}, \bar{v}=0, \bar{T}=T_{w}+A \frac{\partial T}{\partial \bar{y}}, \bar{C}=C_{w}, \bar{N}=-m \frac{\partial \bar{u}}{\partial \bar{y}} \\
& \bar{y} \rightarrow \infty: \bar{u} \rightarrow 0, \bar{N} \rightarrow 0, \bar{T} \rightarrow \infty, \bar{C} \rightarrow \infty,
\end{aligned}
$$

where $\bar{u}$ and $\bar{v}$ are the velocity components in $\bar{x}$ and $\bar{y}$ directions respectively. Also, $\rho, \kappa, \bar{T}, \bar{C}, \bar{N}, B_{o}, \sigma, C_{p}, q_{r}, q^{\prime \prime \prime}, k_{r}$ and $n$ are the fluid density, vortex viscosity, fluid temperature, fluid concentration, component of microrotation, magnetic field intensity, electrical conductivity, specific heat at constant pressure, radiative heat flux, non-uniform heat source/sink, chemical reaction rate, and order of chemical reaction. Also, $\lambda$ is the velocity slip, $A$ is the thermal slip and $m$ is a surface boundary parameter with $0 \leq m \leq 1$. The case when $m=0$ corresponds to $N=0$, this represents no-spin condition i.e. strong concentration such that the micro-particles close to the wall are unable to rotate. 
The case $m=\frac{1}{2}$, indicates weak concentration of micro-particles and the vanishing of anti-symmetric part of the stress tensor and the case $n=1$ represents turbulent boundary layer flows (see Peddieson [29]; Ahmadi, [6]; Jena and Mathur [30]).

$$
\gamma=\left(\mu+\frac{\kappa}{2}\right) j \text {, is the spin gradient viscosity which denotes the relationship }
$$

between the coefficients of viscosity $(\mu)$ and micro-inertia $(j)$. This assumption has been invoked to allow the field of Equations (1)-(5) to predict the correct behaviour in the limiting case when the microstructure effects becomes negligible and the total spin $N$ reduces to the angular velocity (Ahmadi [6]). All the material constants $\mu, \kappa, \gamma, j$ are non-negative.

Using Rosseland approximation,

$$
q_{r}=-\frac{4 \sigma^{\star}}{3 \alpha^{\star}} \frac{\partial T^{4}}{\partial y}
$$

is the radiative heat flux (Adeniyan [31]; Akinbobola and Okoya [32]).

Assuming that there exists sufficiently small temperature difference within the flow such that $T^{4}$ can be expressed as a linear combination of the temperature. Expanding $T^{4}$ in Taylor series about $T_{\infty}$ to get

$$
T^{4}=T_{\infty}^{4}+4 T_{\infty}^{3}\left(T-T_{\infty}\right)+6 T_{\infty}^{2}\left(T-T_{\infty}\right)^{2}+\cdots,
$$

neglecting higher order terms in Equation (8) gives

$$
T^{4}=4 T_{\infty}^{3} T-3 T_{\infty}^{4},
$$

hence

$$
\frac{\partial q_{r}}{\partial \bar{y}}=-\frac{16 \sigma^{\star} T_{\infty}^{3}}{3 \alpha^{\star}} \frac{\partial T^{2}}{\partial \bar{y}^{2}}
$$

The viscosity temperature dependence is assumed to decrease with the absolute temperature in the following form:

$$
\begin{aligned}
\mu(\bar{T}) & =\frac{\mu_{0}}{\left[1+\beta_{\star}\left(\bar{T}-T_{\infty}\right)\right]}=\mu_{0}\left[1-\beta_{\star}\left(\bar{T}-T_{\infty}\right)+\beta_{\star}^{2}\left(\bar{T}-T_{\infty}\right)^{2}+\cdots\right] \\
& \approx \mu_{0}\left[1-\beta_{\star}\left(\bar{T}-T_{\infty}\right)\right]
\end{aligned}
$$

This dependency is in agreement with Batchelor [33], herein $\mu_{0}=\rho v_{0}$ is the fluid viscosity at reference temperature, $v_{0}$ is reference kinematic viscosity; and the coefficient $\beta_{\star}$ with inverse temperature dimension determines the strength of the dependency between $\mu$ and $\bar{T}$.

The non-uniform heat source/sink is given Das [16] as

$$
q^{\prime \prime \prime}=\frac{k u_{w}}{v_{0} \bar{x}}\left[Q\left(T_{w}-T_{\infty}\right) \mathrm{e}^{-\eta}+B\left(T-T_{\infty}\right)\right],
$$

where $Q$ and $B$ are coefficients of space and temperature dependent heat source/sink respectively. The case $Q>0$ and $B>0$ corresponds to internal 
heat generation while $Q<0$ and $B<0$ corresponds to internal heat absorption.

The following similarity transformations are also introduced.

$$
\eta=\left(\frac{a}{v_{0}}\right)^{\frac{1}{2}} y, \psi=\left(a v_{0}\right)^{\frac{1}{2}} \bar{x} f(\eta), \bar{N}=\left(\frac{a}{v_{0}}\right)^{\frac{1}{2}} a \bar{x} g(\eta),
$$

where $\psi$ is the stream function defined as $u=\frac{\partial \psi}{\partial y}, v=-\frac{\partial \psi}{\partial x}$ which identically satisfies Equation (1). Hence

$$
\bar{u}=a \bar{x} f^{\prime}(\eta), \bar{v}=-\left(a v_{0}\right)^{\frac{1}{2}} f(\eta) .
$$

The dimensionless temperature and concentration are given as

$$
\theta=\frac{\bar{T}-T_{\infty}}{T_{w}-T_{\infty}}, \phi=\frac{\bar{C}-C_{\infty}}{C_{w}-C_{\infty}} .
$$

Substituting Equation (13) and Equation (14) in Equations (2)-(6) and using Equations (10)-(12) to obtain

$$
\begin{gathered}
(1+\xi \theta)(1+(1+\xi \theta) K) f^{\prime \prime \prime}+f f^{\prime \prime}-\xi \theta^{\prime} f^{\prime \prime}+(1+\xi \theta)^{2}\left(f f^{\prime \prime}-f^{\prime 2}+K g^{\prime}\right) \\
-(1+\xi \theta)(M(1+\xi \theta)) f^{\prime}=0 \\
(1+K / 2) g^{\prime \prime}+f g^{\prime}-f^{\prime} g-H\left(2 g+f^{\prime \prime}\right)=0 \\
(1+N r) \theta^{\prime \prime}+\operatorname{Prf} \theta^{\prime}+\left(Q \mathrm{e}^{-\eta}+B \theta\right)=0 \\
\phi^{\prime \prime}+S c f \phi^{\prime}-S c \zeta \phi^{\prime \prime}=0 .
\end{gathered}
$$

The boundary conditions become:

$$
\begin{aligned}
& \eta=0: f^{\prime}=1+\alpha f^{\prime \prime}, f=0, g=-m f^{\prime \prime}, \theta=1+\beta \theta^{\prime}, \phi=1 \\
& \eta \rightarrow \infty: f^{\prime}=0, g \rightarrow 0, \theta \rightarrow 0, \phi \rightarrow 0
\end{aligned}
$$

Here, prime denotes differentiation with respect to $\eta, \xi=\beta_{\star}\left(T_{w}-T_{\infty}\right)$ is the variable viscosity parameter, $K=\frac{\kappa}{\mu_{0}}$ is the material parameter, $\alpha=\lambda \sqrt{\frac{a}{v}}$ is the velocity slip parameter, $\beta=A \sqrt{\frac{a}{v}}$ is the thermal slip parameter, $\operatorname{Pr}=\frac{\mu_{0} C_{p}}{k}$ is the Prandtl number, $N r=\frac{16 \sigma^{\star} T_{\infty}^{3}}{3 \alpha^{\star} k}$ is the radiation parameter, $M=\frac{\sigma B_{0}^{2}}{a \rho}$ is the magnetic field parameter, $H=\frac{\kappa}{a \rho j}$ is the vortex viscosity parameter, $S c=\frac{D m}{v}$ is the Schmidt number and $\zeta=\frac{k_{r}\left(C_{w}-C_{\infty}\right)^{n-1}}{a}$ is reaction rate parameter.

\section{Physical Quantities of Engineering Interest}

The physical quantities of engineering interest in this study are: the non-dimensional skin friction, rate of heat transfer and the wall couple stress. 
These are respectively defined as:

$$
C_{f}=\frac{\tau_{w}}{\rho u_{w}^{2}}, N u=\frac{x q_{w}}{k\left(T_{w}-T_{\infty}\right)}, S h=\frac{x q_{m}}{\operatorname{Dm}\left(C_{w}-C_{\infty}\right)}, C s=\frac{x M_{w}}{\mu j a},
$$

where

$$
\begin{aligned}
& \tau_{w}=\left[(\mu+\kappa) \frac{\partial u}{\partial y}+\kappa N\right]_{y=0}, q_{w}=-k\left(\frac{\partial \bar{T}}{\partial y}\right)_{y=0}, \\
& q_{m}=-D m\left(\frac{\partial \bar{C}}{\partial \bar{y}}\right)_{y=0}, M_{w}=\left[\gamma \frac{\partial N}{\partial y}\right]_{y=0},
\end{aligned}
$$

are the wall shear stress, the heat flux, mass flux and the wall couple stress respectively.

In dimensionless form the skin friction, Nusselt number, Sherwood number and wall couple stress correspondingly become

$$
\begin{aligned}
& \left(R e_{x}\right)^{\frac{1}{2}} C_{f}=\frac{1}{1+\xi}(1+K / 2) f^{\prime \prime}(0),\left(R e_{x}\right)^{-\frac{1}{2}} N u_{x}=-\theta^{\prime}(0) \\
& \left(R e_{x}\right)^{-\frac{1}{2}} S h_{x}=-\phi^{\prime}(0),\left(R e_{x}\right)^{-\frac{1}{2}}, \operatorname{Re}_{x} C_{s}=(1+K / 2) g^{\prime}(0),
\end{aligned}
$$

where $\operatorname{Re}_{x}=\frac{u_{w} x}{v}$ is the local Reynolds number.

\section{Method of Solution}

The coupled nonlinear differential Equations (16)-(19) together with the boundary conditions (20) is constitutes a boundary value problem (BVP) which are solved using shooting method alongside fourth order Runge-Kutta method. The higher order nonlinear Equations (16)-(19) of third order in $f$, and second order in $g, \theta$ and $\phi$ are reduced into a system of nine simultaneous equations of first order for nine unknowns. To solve this system, nine initial conditions are needed while only five initial conditions are available. Thus, there are still four initial conditions that are needed which are not given in the problem, these are: $f^{\prime \prime}(0), g^{\prime}(0), \theta^{\prime}(0)$ and $\phi^{\prime}(0)$. However, the values of $f^{\prime}, g, \theta$ and $\phi$ are known as $\eta \rightarrow \infty$. These four end conditions are used to produce the four unknown initial conditions $\left(p_{1}, p_{2}, p_{3}, p_{4}\right)$ at $\eta=0$ by applying the shooting technique. To estimate the value of $\eta_{\infty}$ we start with some initial guess value and solve the BVP Equations (16)-(19) to get $f^{\prime \prime}(0), g^{\prime}(0), \theta^{\prime}(0)$ and $\phi^{\prime}(0)$. The procedure is repeated until two successive values of $f^{\prime \prime}(0), g^{\prime}(0), \theta^{\prime}(0)$, $\phi^{\prime}(0)$ differ only after desired significant digit signifying the limit of the boundary along $\eta$. The last value of $\eta$ is chosen as appropriate for a particular set of governing parameters for the determination of the dimensionless velocity $f^{\prime}(\eta)$, microrotation $g(\eta)$, temperature $\theta(\eta)$ and concentration $\phi(\eta)$ across the boundary layer. The higher order equations are reduced to a system of first order differential equations by letting:

$$
f_{1}=f, f_{2}=f^{\prime}, f_{3}=f^{\prime \prime}, f_{4}=g, f_{5}=g^{\prime}, f_{6}=\theta, f_{7}=\theta^{\prime}, f_{8}=\phi, f_{9}=\phi^{\prime}
$$




$$
\begin{gathered}
f_{3}^{\prime}=\frac{\xi f_{7} f_{3}-f_{1} f_{3}-\left(1+\xi f_{6}\right)^{2}\left(f_{1} f_{3}-f_{2}^{2}+K f_{5}\right)+\left(1+\xi f_{6}\right)\left(M\left(1+\xi f_{6}\right)\right) f_{2}}{\left(1+\xi f_{6}\right)\left(1+\left(1+\xi f_{6}\right) K\right)} \\
f_{5}^{\prime}=\frac{f_{2} f_{4}+H\left(2 f_{4}+f_{3}\right)-f_{1} f_{5}}{(1+K / 2)} \\
f_{7}^{\prime}=\frac{\operatorname{Prf}_{1} f_{7}-\left(Q \mathrm{e}^{-\eta}+B f_{6}\right)}{(1+N r)} \\
f_{9}^{\prime}=S c \zeta f_{8}^{n}-S c f_{1} f_{9} .
\end{gathered}
$$

The boundary conditions now become

$$
\begin{aligned}
& f_{1}(0)=0, f_{2}(0)=1+\alpha f_{3}(0), f_{3}(0)=p_{1}, f_{4}(0)=-n f_{3}(0), \\
& f_{5}(0)=p_{2}, f_{6}(0)=1+\beta f_{7}(0), f_{7}(0)=p_{3}, f_{8}(0)=1, \\
& f_{9}(0)=p_{4}, f_{2}(\infty) \rightarrow 0, f_{4}(\infty) \rightarrow 0, f_{6}(\infty) \rightarrow 0, f_{8}(\infty) \rightarrow 0
\end{aligned}
$$

Thereafter, after gotten all the initial conditions, fourth-order Runge-Kutta integration scheme with step size $\nabla \eta=0.05$ is applied and the solution is obtained with a tolerance limit of $10^{-7}$. The computations are carried out by a commercial package.

\section{Results and Discussion}

To have clear insight into the behaviour of the fluid flow, a computational analysis has been carried out for the dimensionless velocity, temperature, concentration and microrotation profiles across the boundary layer. The default values adopted for computation in this study are: $K=\beta=1, \alpha=0.3, R=0.1$, $Q=0.01, B=0.02, \zeta=M=\xi=0.5, S c=0.62, n=2$ and $\operatorname{Pr}=1.0$. The plotted figures correspond to these values unless otherwise indicated on the graph.

In order to authenticate our numerical analysis, we have compared the values of the local Nusselt number $-\theta(0)$ with the existing works of Grubka and Bobba [34], Chen [35], Seddeek and Salem [36] under some limiting cases for various values of Prandtl number Pr. The comparison shows an excellent agreement as presented in Table 1.

In a similar manner, the computational values of the skin friction coefficient $-f^{\prime \prime}(0)$ compared favourably well with the published works of Anderson [26], Bhattacharyya et al. [23] and Nandeppanavar et al. [37] for variation of the velocity slip parameter $\alpha$ under some limiting cases as displayed in Table 2 .

Furthermore, from the numerical computation, the values of the skin friction coefficient $f^{\prime \prime}(0)$, the Nusselt number $-\theta^{\prime}(0)$, the wall couple stress $-g(0)$, and the Sherwood number $-\phi(0)$ for some selected parameters are sorted out and presented in Table 3. These are: the material parameter $K$, magnetic field parameter $M$, velocity slip parameter $\alpha$, the thermal slip parametr $\beta$, the viscosity parameter $\xi$, order of chemical reaction parameter $n$, and the rate of reaction parameter $\zeta$. From this table, it is observed that the skin friction coefficient $f^{\prime \prime}(0)$ 
Table 1. Comparison of the local Nusselt number $-\theta^{\prime}(0)$ for various values of $\operatorname{Pr}$ with $M=K=A=B=\xi=S c=R=\alpha=\beta=0$.

\begin{tabular}{ccccc}
\hline$P r$ & Grubka \& Bobba [34] & Chen [35] & Seddek \& Salem [36] & Present Results \\
\hline 0.72 & 0.4631 & 0.46315 & 0.46314 & 0.463157 \\
1.00 & 0.5820 & 0.58199 & 0.58197 & 0.581977 \\
3.00 & 1.1652 & 1.16523 & 1.16524 & 1.165246 \\
7.00 & - & 1.89537 & 1.89540 & 1.895403 \\
10.00 & 2.3080 & 2.30797 & 2.30800 & 2.308004 \\
\hline
\end{tabular}

Table 2. Comparison of the skin friction coefficient $-f^{\prime \prime}(0)$ for various values of $\alpha$ when $M=K=A=B=\xi=S C=R=\beta=0$.

\begin{tabular}{ccccc}
\hline$\alpha$ & Anderson [26] & Bhattacharyya et al. [23] & Nandeppanavar et al. [37] & Present Results \\
\hline 0.1 & 0.8721 & 0.872083 & 0.872080 & 0.872083 \\
0.2 & 0.7764 & 0.776377 & 0.776380 & 0.776377 \\
0.5 & 0.5912 & 0.591195 & 0.591200 & 0.591196 \\
5.0 & 0.1448 & 0.144840 & 0.144840 & 0.144842 \\
10.0 & 0.0812 & 0.081242 & 0.081240 & 0.081245 \\
20.0 & 0.0438 & 0.043789 & 0.043790 & 0.043792 \\
50.0 & 0.0186 & 0.018597 & 0.018600 & 0.018600 \\
\hline
\end{tabular}

Table 3. Values of skin friction coefficient, Nusselt number, wall couple stress and Sherwood number for variation in $K, M, \alpha, \beta, \xi, n$ and $\zeta$.

\begin{tabular}{ccccccccccc}
\hline$K$ & $M$ & $\alpha$ & $\beta$ & $\xi$ & $n$ & $\zeta$ & $-f^{\prime \prime}(0)$ & $-\theta^{\prime}(0)$ & $-g^{\prime}(0)$ & $-\phi^{\prime}(0)$ \\
\hline 0.0 & 0.5 & 0.3 & 1.0 & 0.5 & 2.0 & 0.5 & 0.942679 & 0.248165 & 0.426310 & 0.548686 \\
1.0 & 0.5 & 0.3 & 1.0 & 0.5 & 2.0 & 0.5 & 0.771714 & 0.288348 & 0.286937 & 0.574186 \\
2.5 & 0.5 & 0.3 & 1.0 & 0.5 & 2.0 & 0.5 & 0.642858 & 0.318552 & 0.197571 & 0.599941 \\
1.0 & 0.5 & 0.3 & 1.0 & 0.5 & 2.0 & 0.5 & 0.728596 & 0.300300 & 0.271118 & 0.583413 \\
1.0 & 0.75 & 0.3 & 1.0 & 0.5 & 2.0 & 0.5 & 0.819484 & 0.274335 & 0.304522 & 0.564700 \\
1.0 & 1.2 & 0.3 & 1.0 & 0.5 & 2.0 & 0.5 & 0.893051 & 0.251233 & 0.331703 & 0.551605 \\
1.0 & 0.5 & 0.0 & 1.0 & 0.5 & 2.0 & 0.5 & 1.089992 & 0.325681 & 0.453464 & 0.609351 \\
1.0 & 0.5 & 0.7 & 1.0 & 0.5 & 2.0 & 0.5 & 0.773435 & 0.228877 & 0.287144 & 0.573414 \\
1.0 & 0.5 & 1.2 & 1.0 & 0.5 & 2.0 & 0.5 & 0.567015 & 0.197786 & 0.191007 & 0.547994 \\
1.0 & 0.5 & 0.3 & 0.0 & 0.5 & 2.0 & 0.5 & 0.787782 & 0.399718 & 0.293049 & 0.571112 \\
1.0 & 0.5 & 0.3 & 1.0 & 0.5 & 2.0 & 0.5 & 0.773435 & 0.228877 & 0.287143 & 0.573414 \\
1.0 & 0.5 & 0.3 & 2.0 & 0.5 & 2.0 & 0.5 & 0.764817 & 0.187235 & 0.283884 & 0.575107 \\
1.0 & 0.5 & 0.3 & 1.0 & 0.0 & 2.0 & 0.5 & 0.265482 & 0.301152 & 0.265482 & 0.584242 \\
1.0 & 0.5 & 0.3 & 1.0 & 0.3 & 2.0 & 0.5 & 0.279788 & 0.234221 & 0.279788 & 0.577214 \\
1.0 & 0.5 & 0.3 & 1.0 & 1.0 & 2.0 & 0.5 & 0.300690 & 0.217631 & 0.300690 & 0.566062 \\
1.0 & 0.5 & 0.3 & 1.0 & 0.5 & 1.0 & 0.5 & 0.771714 & 0.288348 & 0.286937 & 0.677426 \\
1.0 & 0.5 & 0.3 & 1.0 & 0.5 & 2.0 & 0.5 & 0.771714 & 0.288348 & 0.286937 & 0.574186 \\
1.0 & 0.5 & 0.3 & 1.0 & 0.5 & 3.0 & 0.5 & 0.771714 & 0.288348 & 0.286937 & 0.519564 \\
1.0 & 0.5 & 0.3 & 1.0 & 0.5 & 0.5 & 0.0 & 0.771714 & 0.288348 & 0.286937 & 0.338616 \\
1.0 & 0.5 & 0.3 & 1.0 & 0.5 & 0.5 & 0.5 & 0.771714 & 0.288348 & 0.286937 & 0.574186 \\
\hline & & & & & & & & & & \\
\hline
\end{tabular}


decreases with an increase in $K, \alpha$ and $\beta$ while it increases with $M$ and $\xi$. The local Nusselt number $-\theta^{\prime}(0)$ decreases for $M, \alpha, \beta$ and $\xi$ but increases for a rise in $K$. A rise in $K, \alpha, \beta$ and $\xi$ reduces the wall couple stress while a rise in parameter $M$ enhances it. The mass transfer rate increases with a rise in $K$ while it falls with a rise in $\alpha$.

Figures 2-5 exhibit the effects of the velocity slip parameter $\alpha$ on the velocity, temperature, concentration and microrotation profiles across the boundary layer. There is a decrease in the fluid velocity and the microrotation profiles as $\alpha$ increases as shown in Figure 2 and Figure 5. Moreover, it is observed that the rate of transport reduces with the increasing distance $(\eta)$ from the sheet for the velocity and microrotation profiles. In the presence of slip, the stretching velocity and the flow velocity near the sheet are unequal. Therefore, an increase in the slip parameter $\alpha$ causes a rise in the slip velocity leading to a decrease in the fluid velocity as observed in Figure 2. The temperature and concentration

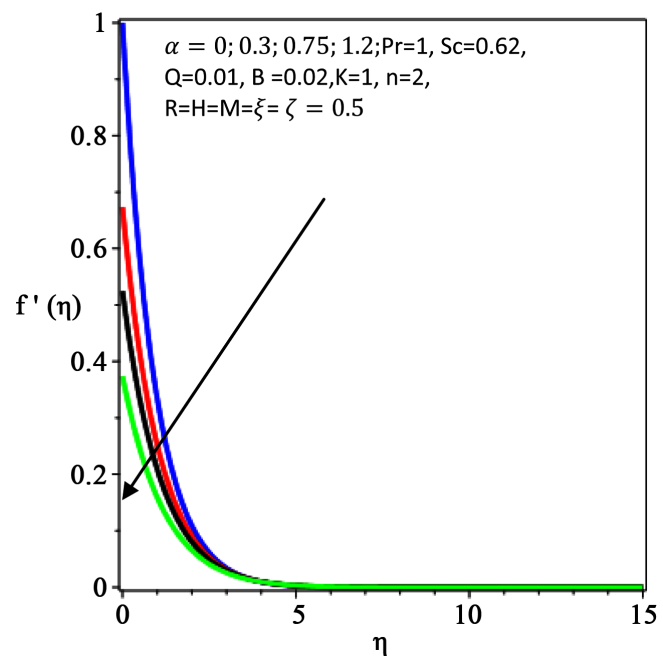

Figure 2. Effect of $\alpha$ on velocity profiles.

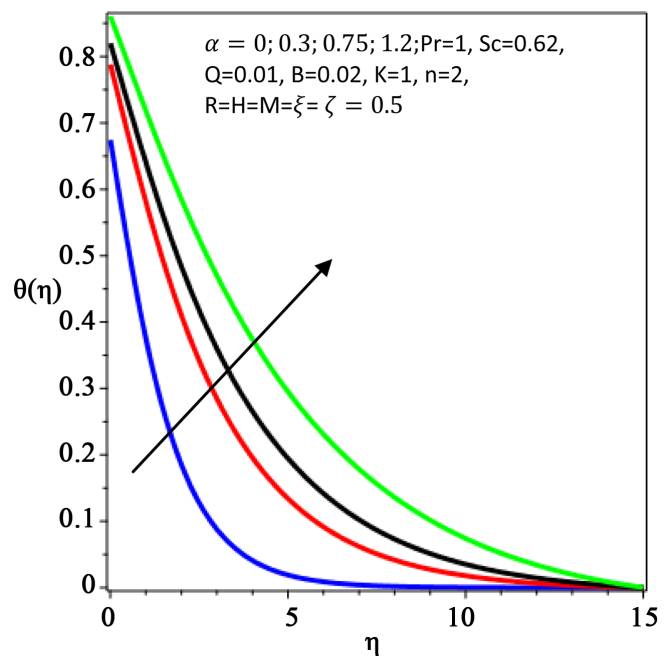

Figure 3. Effect of $\alpha$ on temp. profiles. 


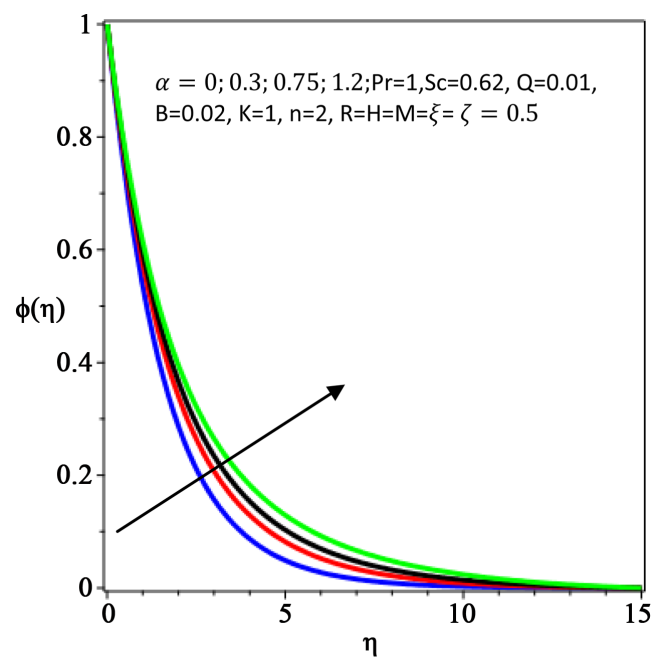

Figure 4. Effect of $\alpha$ on concentration.

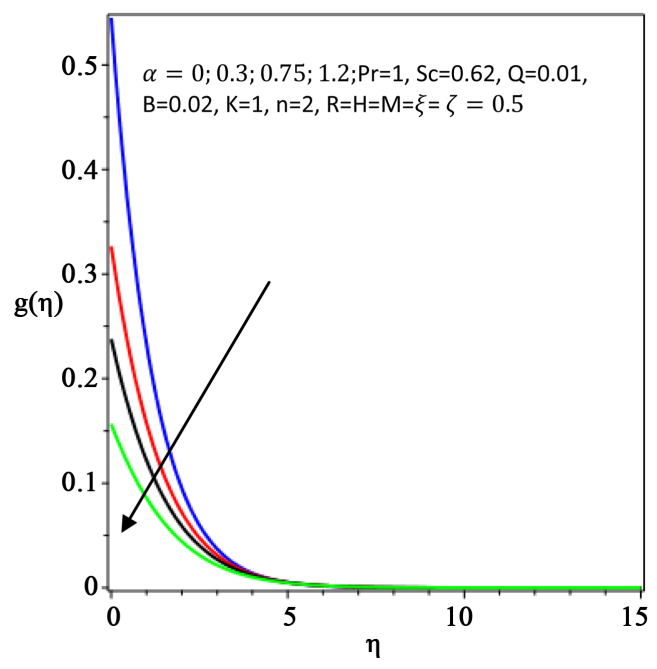

Figure 5. Effect of $\alpha$ on microrotation.

profiles are enhanced with an increase in the velocity slip parameter $\alpha$ as seen in Figure 3 and Figure 4. In addition, an increase in $\alpha$ causes the thickening of the thermal and the solutal boundary layer thicknesses.

The influence of the thermal slip parameter $\beta$ on the temperature profiles is illustrated in Figure 6. It is noticed that the temperature decreases near the sheet with an increase in $\beta$. This response is due to the fact that as $\beta$ increases, less heat is transferred from the sheet to the fluid leading to a drop in the temperature. (see Table 3). Figure 7 shows that a rise in the magnetic field parameter $M$ reduces the fluid velocity. An increase in the transverse magnetic field in an electrically conducting fluid has the tendency to increase the resistive force known as Lorentz force and thus reduces the fluid motion and the hydrodynamic boundary layer thickness.

Figures 8-11 describe the effect of the material parameter $K$ on the velocity, temperature, concentration and microrotation profiles. Evidently, there is a rise 


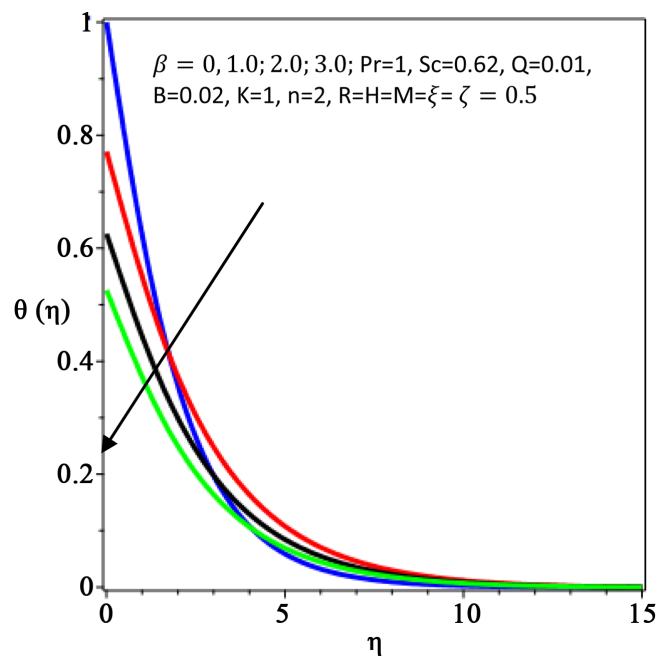

Figure 6. Effect of $\beta$ on temperature.

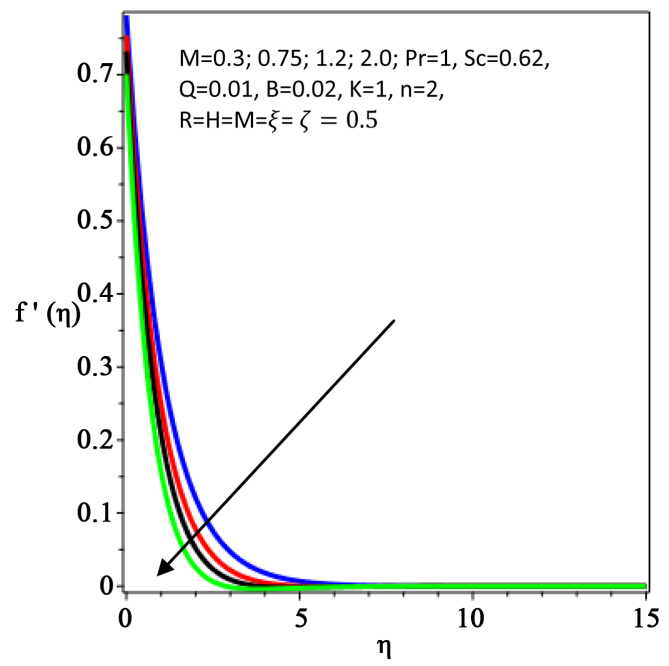

Figure 7. Effect of $M$ on velocity.

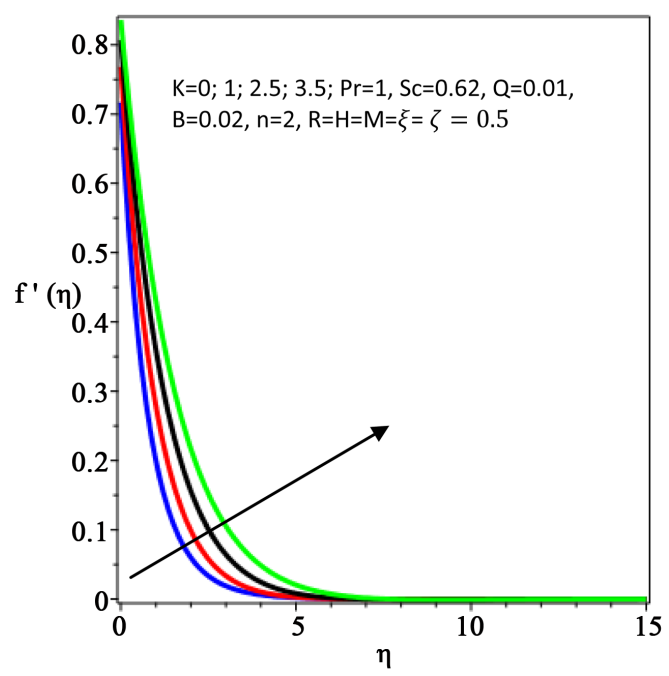

Figure 8. Effect of $K$ on velocity profiles. 


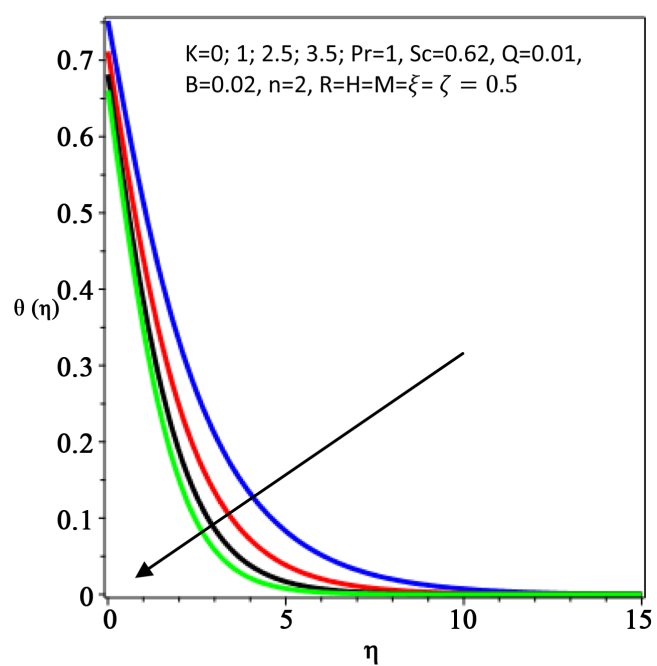

Figure 9. Effect of $K$ on temperature.

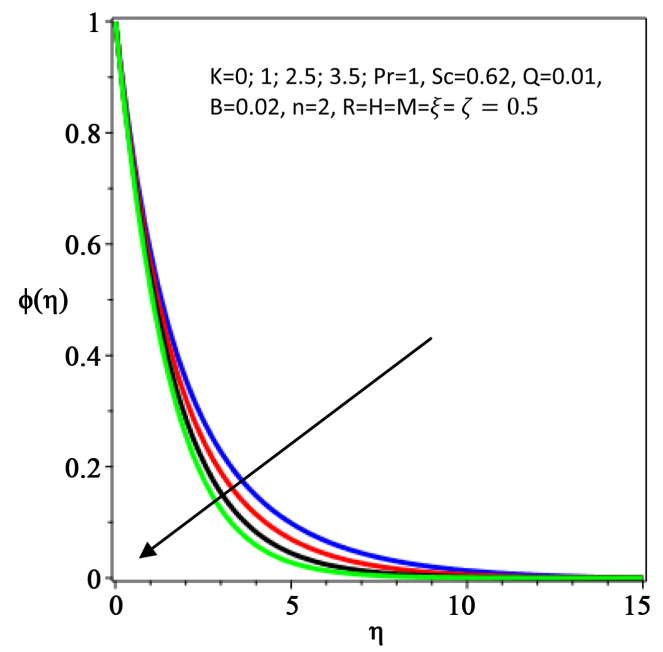

Figure 10. Effect of $K$ on concentration profiles.

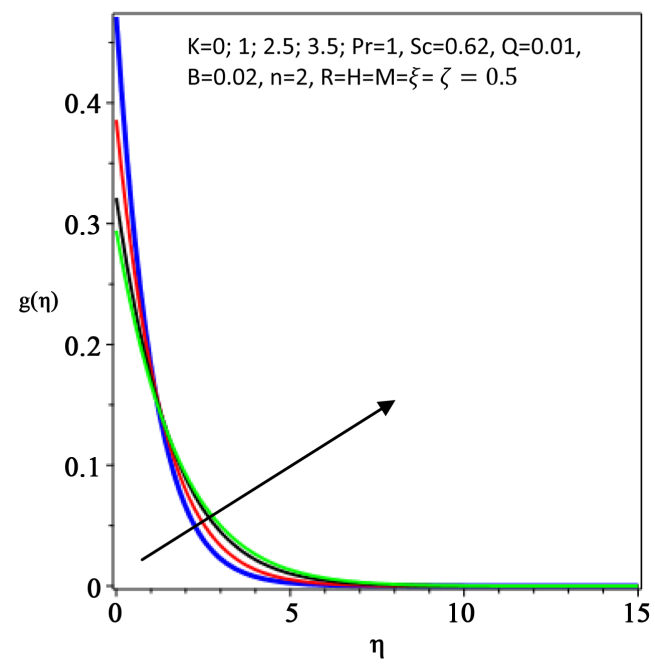

Figure 11. Effect of $K$ on microrotation. 
in the velocity profiles as $K$ increases due to the thickening of the hydrodynamic boundary layer. However, the temperature and concentration profiles with the thermal and the solutal boundary layer thicknesses decrease with an increase in the material parameter $K$ as displayed in Figure 9 and Figure 10. Moreover, the effect of the material parameter $K$ on the microrotation profiles is depicted in Figure 11. Observation reveals that there is a decrease in the microrotation distribution near the sheet as $K$ increases, whereas as the distance $(\eta)$ from the sheet increases, the profiles intersect and the opposite trend is noticed, i.e. the microrotation profiles rise with an increase in $K$.

Figures 12-14 illustrate the effect of the variable viscosity parameter $\xi$ on the velocity, temperature and concentration profiles. Clearly, an increase in $\xi$ results in reducing the fluid motion and the thinning of the hydrodynamic boundary layer thickness as depicted in Figure 12. This response is due to the fact that a rise in the viscosity parameter enhances the skin friction coefficient (see Table 3)

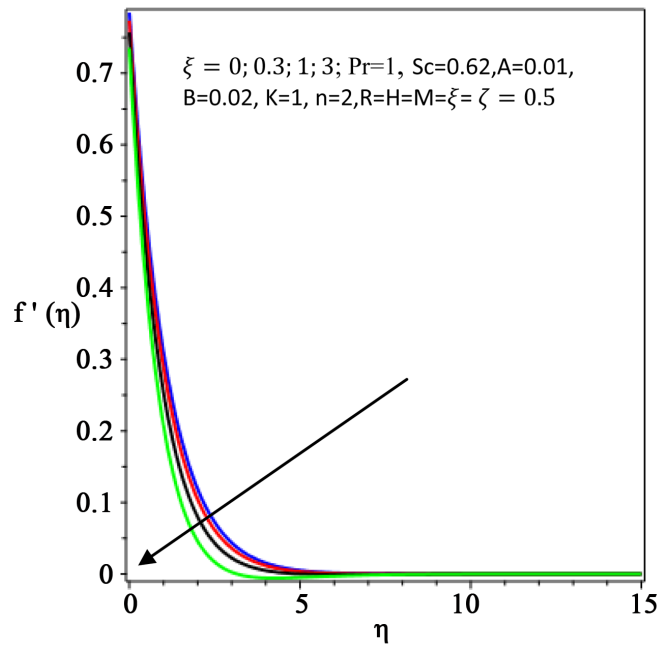

Figure 12. Effect of $\xi$ on velocity profiles.

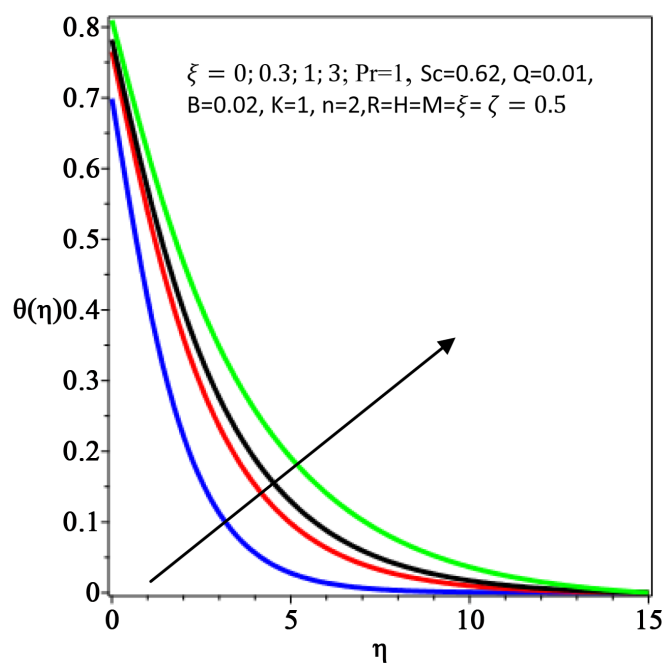

Figure 13. Effect of $\xi$ on temperature. 


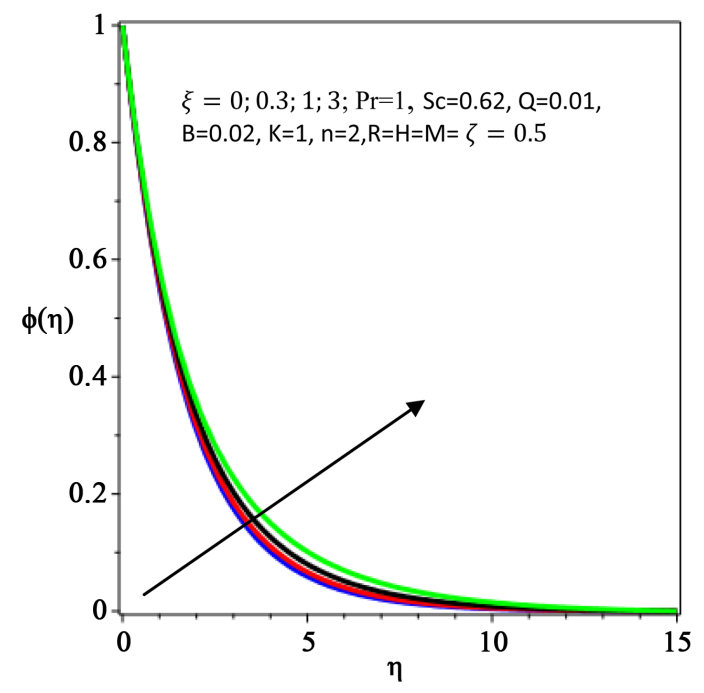

Figure 14. Effect of $\xi$ on concentration.

thus lowering the fluid velocity. As an increase in the variable viscosity parameter $\xi$ causes a rise in the skin friction, the area of the stretching surface in contact with the flow rises and more heat is generated leading to a rise in the surface temperature and the thickening of the thermal boundary layer thickness as demonstrated in Figure 13. A similar trend occurs for the concentration profiles as described in Figure 14. However, the increase is more pronounced in the temperature profiles than that of concentration profiles. In addition, it is noticed that as $\xi$ increases, the skin friction coefficient rises whereas the Nusselt number and the wall couple stress fall as seen on Table 3. This agrees well with Das [16].

Figure 15 depicts the influence of the homogeneous chemical reaction parameter $\zeta$ on the concentration profiles. An increase in $\zeta$ causes a decrease in the concentration of the micropolar fluid flow along the sheet due to the thinning of the solutal boundary layer thickness. In contrast, an increase in the order of the chemical reaction $n$ enhances the concentration profiles as demonstrated in Figure 16. Also, an increase in $\zeta$ lowers the mass transfer rate while there is a rise in the mass transfer in response to higher values of reaction order $n$ as shown in Table 3.

The influences of the space and heat dependent heat source parameters $Q>0, B>0$ on the temperature profiles are displayed in Figure 17 and Figure 18. It is clear from these Figures that the thermal boundary layer thickness increases with an increase in the magnitude of both $Q>0$ and $B>0$. This is due to the fact that energy is generated by the imposition of $Q>0$ and $B>0$ leading to an increase in the micropolar fluid temperature, thereby facilitating an increase in the temperature profiles. Figure 19 describes the effect of the vortex viscosity parameter $H$ on the microrotation profiles. It is noticed that the microrotation profiles reduce with an increase in $H$. The microrotation boundary layer thickness also decreases with an increase in the vortex viscosity parameter $H$. 


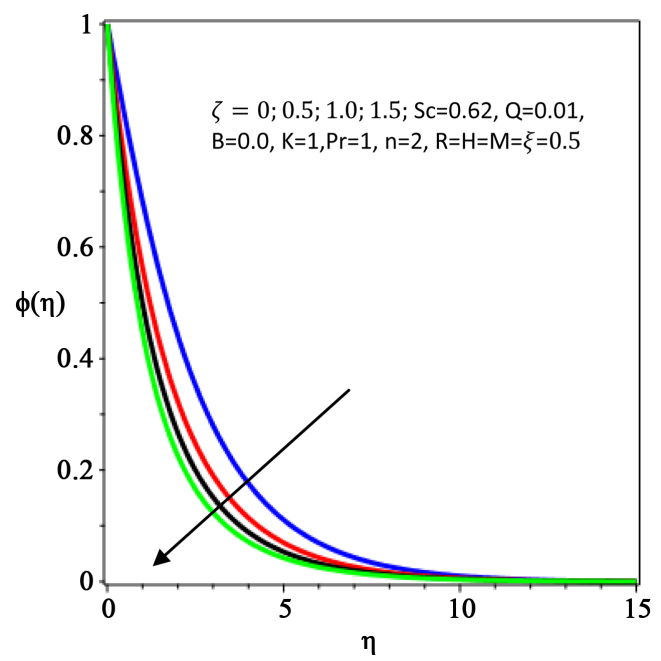

Figure 15. Effect of $\zeta$ on concentration.

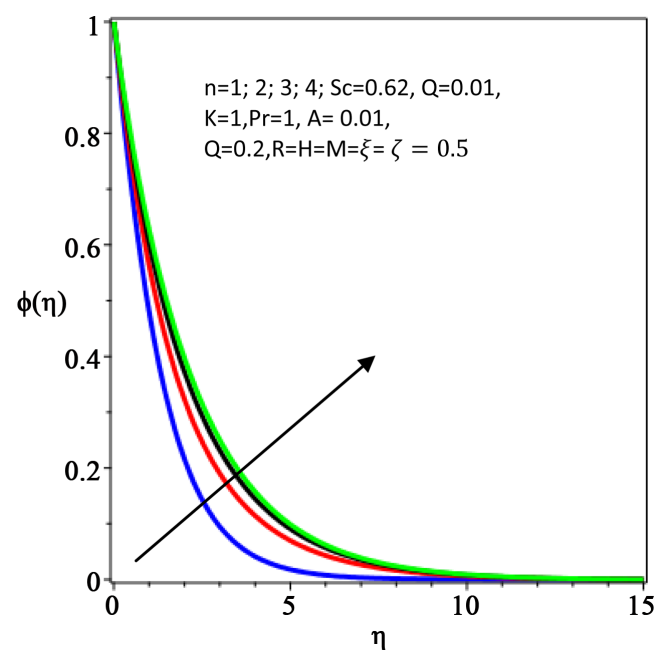

Figure 16. Effect of $n$ on concentration.

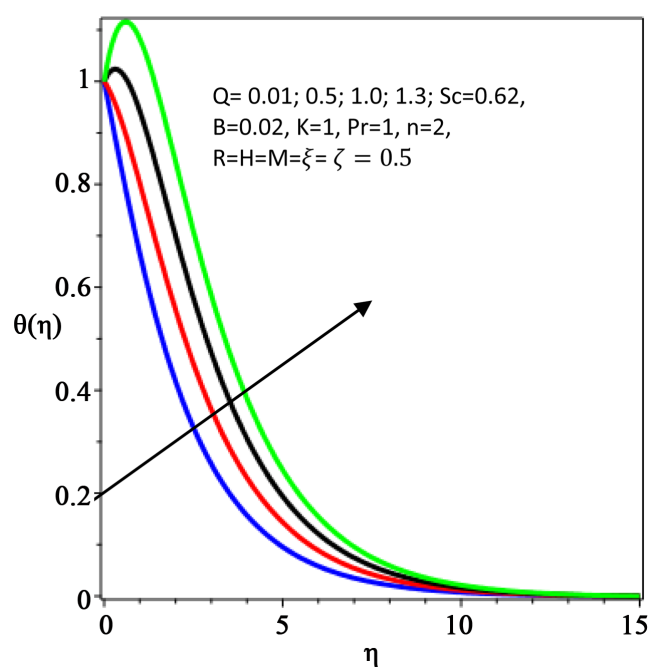

Figure 17. Effect of $Q$ on temperature. 


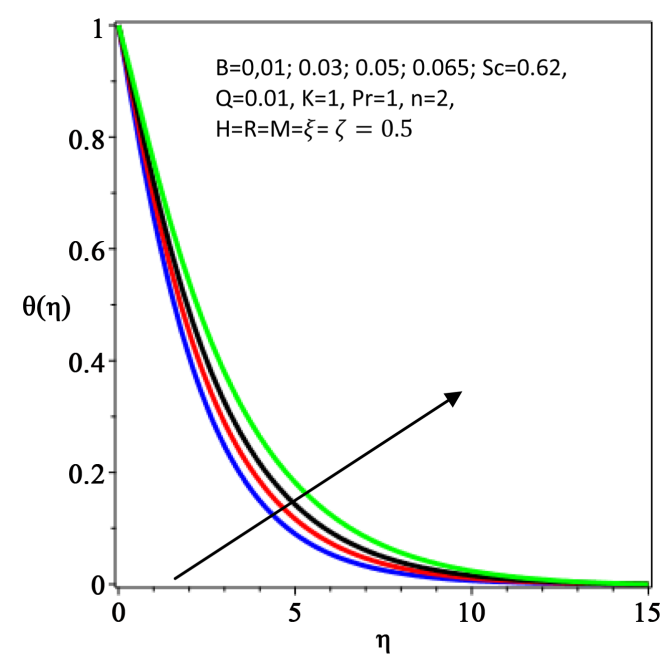

Figure 18. Effect of $B$ on temperature profiles.

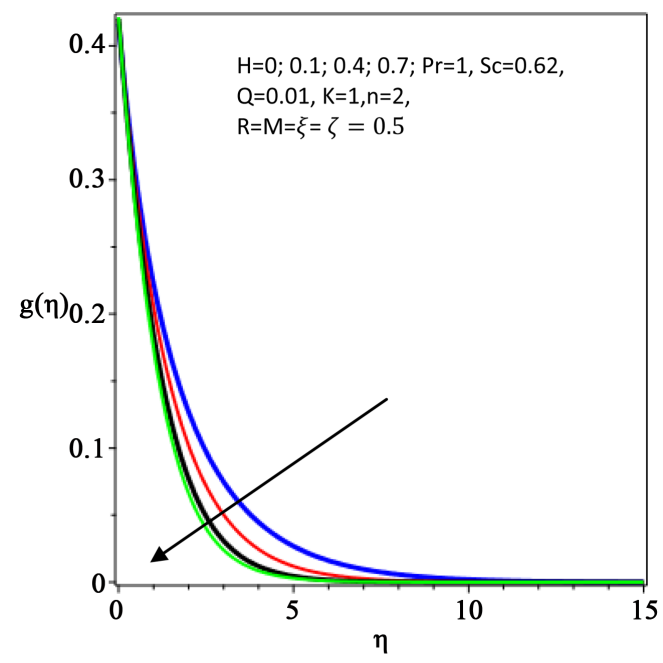

Figure 19. Effect of $H$ on microrotation.

\section{Conclusions}

This study has investigated heat and mass transfer flow in an electrically conducting micropolar fluid over a stretching sheet with the effects of velocity and thermal slip conditions in the presence of temperature dependent viscosity. The effects of thermal radiation, non-uniform heat generation/absorption and chemical reaction of a general order have also been considered on the problem. The resulting governing equations are numerically solved by shooting method coupled with fourth order Runge-Kutta integration scheme and the influences of the emerging physical parameters are presented through graphs and tables. Also, the present results compared well with the existing results in literature for some limiting cases. It is observed that:

- An increase in the material parameter $K$, velocity slip parameter $\alpha$ and the thermal slip parameter $\beta$ reduce the skin friction coefficient $f^{\prime \prime}(0)$ while the opposite trend is the case with the magnetic and viscosity parameters $M$ and 
$\xi$ respectively.

- The influences of the magnetic parameter $M$, velocity slip $\alpha$, thermal slip $\beta$ and viscosity parameters $\xi$ are to decrease the local Nusselt number $-\theta^{\prime}(0)$ whereas an increase in the material parameter enhances both the heat and mass transfers.

- The effect of the velocity slip is to reduce the velocity and microrotation profiles while the temperature profile is enhanced. However, the temperature profile falls with an increase in the thermal slip parameter.

- A rise in the velocity profiles is observed as the material parameter $K$ increases due to the thickening of the hydrodynamic boundary layer. However, the temperature and concentration profiles decrease with an increase in the material parameter $K$.

- The microrotation distribution in the boundary layer reduces with a rise in the vortex viscosity parameter $H$ whereas it increases as the material parameter $K$ rises.

- An increase in the rate of the chemical reaction causes a decrease in the species concentration of the micropolar fluid flow whereas an increase in the order of the chemical reaction enhances the concentration profiles.

\section{Acknowledgements}

The authors would like to acknowledge the reviewers and the editor for their useful suggestions and contributions which led to the improvement of this paper.

\section{References}

[1] Chen, J., Liang, C. and Lee, J.D. (2011) Theory and Simulation of Micropolar Fluid Dynamics. Journal of Nanoengineering and Nanosystems, 224, 31-39.

[2] Hoyt, J.W. and Fabula, A.G. (1964) The Effects of Additives on Fluid Friction. US Naval Ordinance Test Station Report.

[3] Vogel, W.A. and Patterson, A.M. (1964) An Experimental Investigation of Additives Injected into the Boundary Layer of an Underwater Body. Pacific Naval Lab. of the Defense Res. Board of Canada, Report.

[4] Eringen, A.C. (1966) Theory of Micropolar Fluids. Journal of Mathematical Analysis and Applications, 16, 1-18. https://doi.org/10.1512/iumj.1967.16.16001

[5] Eringen, A.C. (1972) Theory of Thermo-Microfluids. Journal of Mathematical Analysis and Applications, 38, 480-496. https://doi.org/10.1016/0022-247X(72)90106-0

[6] Ahmadi, G. (1976) Self-Similar Solution of Incompressible Micropolar Boundary Layer Flow over a Semi-Infinite Plate. International Journal of Engineering Science, 14, 639-646. https://doi.org/10.1016/0020-7225(76)90006-9

[7] Hayat, T., Mustafa, M. and Obaidat, S. (2011) Soret and Dufour Effects on the Stagnation Point Flow of a Micropolar Fluid toward a Stretching Sheet. Journal of Fluid Engineering, 133, 1-9. https://doi.org/10.1115/1.4003505

[8] Rahman, M.M. (2009) Convective Flows of Micropolar Fluids from Radiate Isothermal Porous Surface with Viscous Dissipation and Joule Heating. Communica- 
tions in Nonlinear Science and Numerical Simulation, 14, 3018-3030.

https://doi.org/10.1016/j.cnsns.2008.11.010

[9] Lukaszewicz, G. (1999) Micropolar Fluids: Theory and Applications. Birkhauser, Boston. https://doi.org/10.1007/978-1-4612-0641-5

[10] Crane, L.J. (1970) Flow past a Stretching Plate. Communicatios Breves, 21, 645-647. https://doi.org/10.1007/BF01587695

[11] Gupta, P.S. and Gupta, A.S. (1977) Heat and Mass Transfer on a Stretching Sheet with Suction or Blowing. The Canadian Journal of Chemical Engineering, 55, 744-746. https://doi.org/10.1002/cjce.5450550619

[12] Mukhopadhyay, S. (2013) Effects of Thermal Radiation and Variable Fluid Viscosity on Stagnation Point Flow past a Porous Stretching Sheet. Meccanica, 48, 1717-1730. https://doi.org/10.1007/s11012-013-9704-0

[13] Eldabe, N.T., Elshehawey, E.F., Elbarbary, M.E. and Elgazery, N.S. (2005) Chebyshev Finite Difference Method for MHD Flow of a Micropolar Fluid past a Stretching Sheet with Heat Transfer. Journal of Applied Mathematics and Computation, 160, 437-450. https://doi.org/10.1016/j.amc.2003.11.013

[14] Elbashbeshy, E.M.A. and Bazid, M.A.A. (2004) Heat Transfer in a Porous Medium over a Stretching Surface with Internal Heat Generation and Suction or Injection. Applied Mathematics and Computation, 158, 799-807.

https://doi.org/10.1016/j.amc.2003.08.141

[15] Kumar, L. (2009) Finite Element Analysis of Combined Heat and Mass Transfer in Hydromagnetic Micropolar Flow along a Stretching Sheet. Computational Materials Science, 46, 841-848. https://doi.org/10.1016/j.commatsci.2009.04.021

[16] Das, K. (2012) Slip Effects on Heat and Mass Transfer in MHD Microplar Fluid Flow over an Inclined Plate with Thermal Radiation and Chemical Reaction. International Journal for Numerical Methods in Fluids, 70, 96-113.

[17] Mishra, S.R., Baag, S. and Mohapatra, D.K. (2016) Chemical Reaction and Soret Effects on Hydromagnetic Micropolar Fluid along a Stretching Sheet. Engineering Science and Technology, an International Journal, 19, 1919-1928.

[18] Bhattacharyya, K. and Layek, G.C. (2012) Similarity Solution of MHD Boundary Layer Flow with Diffusion and Chemical Reaction over a Porous Flat Plate with Suction/Blowing. Meccanica, 47, 1043-1048.

https://doi.org/10.1007/s11012-011-9461-x

[19] Mohamed, R.A. and Abo-Dahab, S.M. (2009) Influence of Chemical Reaction and Thermal Radiation on the Heat and Mass Transfer in MHD Micropolar Flow over a Vertical Moving Porous Plate in a Porous Medium with Heat Generation. International Journal of Thermal Sciences, 48, 1800-1813. https://doi.org/10.1016/j.ijthermalsci.2009.01.019

[20] Olajuwon, B.I., Oahimire, J.I. and Waheed, M.A. (2014) Convection Heat and Mass Transfer in a Hydromagnetic Flow of a Micropolar Fluid over a Porous Medium. Theoretical and Applied Mechanics, 41, 93-117. https://doi.org/10.2298/TAM1402093O

[21] Pal, D. and Chatterjee, S. (2010) Heat and Mass Transfer in MHD Non-Darcian Flow of a Micropolar Fluid over a Stretching Sheet Embedded in a Porous Media with Non-Uniform Heat Source and Thermal Radiation. Communications in Nonlinear Science and Numerical Simulation, 15, 1843-1857. https://doi.org/10.1016/j.cnsns.2009.07.024

[22] Pal, D. and Mondal, M. (2014) Effects of Temperature-Dependent Viscosity and Variable Thermal Conductivity on MHD Non = Darcy Mixed Convective Diffusion of Species over a Stretching Sheet. Journal of the Egyptian Mathematical Society, 22, 
123-135. https://doi.org/10.1016/j.joems.2013.05.010

[23] Bhattacharyya, K., Layek, G.C. and Gorla, R.S.D. (2013) Boundary Layer Slip Flow and Heat Transfer past a Stretching Sheet with Temperature Dependent Viscosity. Thermal Energy and Power Engineering, 2, 38-43.

[24] Wang, C.Y. (2002) Flow Due to Stretching Boundary with Partial Slip-An Exact Solution of the Navier Stokes Equation. Chemical Engineering Science, 57, 3745-3747. https://doi.org/10.1016/S0009-2509(02)00267-1

[25] Mukhopadhyay, S. (2012) Slip Effects on MHD Boundary Layer Flow over an Exponentially Stretching Sheet with Suction/Blowing and Thermal Radiation. Ain Shams Engineering Journal, 4, 485-491.

[26] Anderson, H.I. (2002) Sip Flow past a Stretching Surfcade. Acta Mechanica, 158, 121-125. https://doi.org/10.1007/BF01463174

[27] Devi, R.L., Neeraja, A. and Reddy, N.B. (2015) Radiation Effect on MHD Slip Flow past a Stretching Sheet with Variable Viscosity and Heat Source/Sink. International Journal of Scientific and Innovative Mathematical Research, 3, 8-17.

[28] Kemparaju, M.C., Abel, M.C. and Nandeppanavar, M.M. (2015) Heat Transfer in MHD Flow over a Stretching Sheet with Velocity and Thermal Slip Condition. Advances in Physics Theories and Applications, 49, 25-33.

[29] Peddieson, J. (1972) An Application of the Micropolar Model to the Calculation of a Turbulent Shear Flow. International Journal of Engineering Science, 10, 23-32. https://doi.org/10.1016/0020-7225(72)90072-9

[30] Jena, S.K. and Mathur, M.N. (1981) Similarity Solutions for Laminar Free Convection Flow of a Thermomicropolar Fluid past a Non-Isothermal Flat Plate. International Journal of Engineering Science, 19, 1431-1439.

https://doi.org/10.1016/0020-7225(81)90040-9

[31] Adeniyan, A. (2015) MHD Mixed Convection of a Viscous Dissipating and Chemically Reacting Stagnation-Point Flow near a Vertical Permeable Plate in a Porous Medium with Thermal Radiation and Heat Source/Sink. Asian Journal of Mathematics and Applications, 1-23.

[32] Akinbobola, T.E. and Okoya, S.S. (2015) The Flow of Second Grade Fluid over a Stretching Sheet with Variable Thermal Conductivity and Viscosity in the Presence of Heat Source/Sink. Journal of Nigeria Mathematical Society, 34, 331-342. https://doi.org/10.1016/j.jnnms.2015.10.002

[33] Batchelor, G.K. (1987) An Introduction to Fluid Dynamics. Camb. Univ. Press, London, 597.

[34] Grubka, L.K. and Bobba, K.M. (1985) Heat and Mass Transfer Characteristics of a Stretching Surface with Variable Temperature. Transactions of the ASME, 107, 248-250.

[35] Chen, C.H. (1998) Laminar Mixed Convection Adjacent to Vertical Continuously Stretching Sheets. Heat and Mass Transfer, 33, 471-476. https://doi.org/10.1007/s002310050217

[36] Seddeek, M.A. and Salem, A.M. (2005) Laminar Mixed Convection Adjacent to Vertical Continuously Stretching Sheets with Variable Viscosity and Variable Thermal Diffusivity. Heat and Mass Transfer, 41, 1048-1055. https://doi.org/10.1007/s00231-005-0629-6

[37] Nandeppanavar, M.M., Vajravelu, K., Abel, M.S. and Siddalingappa, M.N. (2013) MHD Flow and Heat Transfer over a Stretching Surface with Variable Thermal Conductivity and Partial Slip. Meccanica, 48, 1451-1464. https://doi.org/10.1007/s11012-012-9677-4 